\title{
Simvastatin attenuates neutrophil recruitment in one-lung ventilation model in rats $^{1}$
}

\author{
Camila Ferreira LeiteI, Fábio André Marangoni" ${ }^{I I}$, Enilton Aparecido Camargo ${ }^{\mathrm{III}}$, Angélica de Fátima de Assunção Braga ${ }^{\mathrm{IV}}$, Ivan \\ Felizardo Contrera Torov, Edson Antunes ${ }^{\mathrm{VI}}$, Elen Cristina Tiezem Landucci ${ }^{\mathrm{VII}}$, Ricardo Kalaf Mussi ${ }^{\mathrm{VIII}}$ \\ IFellow Master degree, Postgraduate Program in Sciences of Surgery, Faculty of Medical Sciences, Department of Surgery, UNICAMP, Campinas-SP, \\ Brazil. Acquisition, analysis and interpretation of data, technical procedures, manuscript writing.

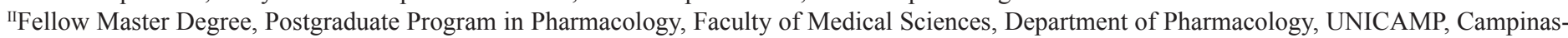 \\ SP, Brazil. Acquisition of data and technical procedures. \\ II'Associate Professor, Department of of Physiology, Centre of Biological and Health Sciences, Federal University of Sergipe (UFS), Sao Cristovao-SE, \\ Brazil. Design the protocol and substantive scientific and intellectual contributions to the study.

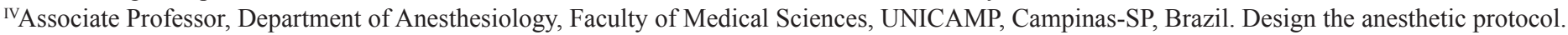 \\ ${ }^{v}$ Associate Professor, Department of Surgery, Faculty of Medical Sciences, UNICAMP, Campinas-SP, Brazil. Substantive scientific and intellectual \\ contributions to the study. \\ ${ }^{\mathrm{V}}$ Full Professor, Department of Pharmacology, Faculty of Medical Sciences, UNICAMP, Campinas-SP, Brazil. Critical revision. \\ ${ }^{V I I}$ Associate Professor, Department of Pharmacology, Faculty of Medical Sciences, UNICAMP, Campinas-SP, Brazil. Conception and design of the \\ study. \\ VIIIProfessor, Postgraduate Program in Sciences of Surgery, Head of Division of Thoracic Surgery, Department of Surgery, Faculty of Medical Sciences, \\ UNICAMP, Campinas-SP, Brazil. Coordinate the trial.
}

\section{ABSTRACT}

PURPOSE: To investigate the anti-inflammatory effects of simvastatin in rats undergoing one-lung ventilation (OLV) followed by lung re-expansion.

METHODS: Male Wistar rats $(\mathrm{n}=30)$ were submitted to $1-\mathrm{h}$ OLV followed by $1-\mathrm{h}$ lung re-expansion. Treated group received simvastatin (40 mg/kg for 21 days) previous to OLV protocol. Control group received no treatment or surgical/ventilation interventions. Measurements of pulmonary myeloperoxidase (MPO) activity, pulmonary protein extravasation, and serum levels of cytokines and C-reactive protein (CRP) were performed.

RESULTS: OLV significantly increased the MPO activity in the collapsed and continuously ventilated lungs (31\% and 52\% increase, respectively) compared with control $(\mathrm{p}<0.05)$. Treatment with simvastatin significantly reduced the MPO activity in the continuously ventilated lung but had no effect on lung edema after OLV. The serum IL-6 and CRP levels were markedly higher in OLV group, but simvastatin treatment failed to affect the production of these inflammatory markers. Serum levels of IL-1 $\beta$, TNF- $\alpha$ and IL-10 remained below the detection limit in all groups.

CONCLUSIONS: In an experimental one-lung ventilation model pre-operative treatment with simvastatin reduces remote neutrophil infiltration in the continuously ventilated lung. Our findings suggest that simvastatin may be of therapeutic value in OLV-induced pulmonary inflammation deserving clinical investigations.

Key words: Models, Animal. Pulmonary Ventilation. Simvastatin. Thoracic Surgery. Inflammation. Rats. 


\section{Introduction}

The one-lung ventilation (OLV), a ventilation strategy used during thoracic surgery, consists in an intentional and temporary collapse of a lung ${ }^{1}$, while the other one remains normally ventilated. This ventilator strategy initiates a series of pathophysiologic events that can be attributed to two major factors, namely hypoxia and re-oxygenation. The cellular damage that follows the oxygen deprivation is exacerbated during the reoxygenation by the generation of free radicals ${ }^{2}$. OLV in animals has been accepted as an ideal model to produce lung injury associated with organ failure and high mortality levels ${ }^{3}$. The control of the pulmonary inflammation resulting from thoracic surgical manipulations has been a great challenge. Although some pharmacological treatments aiming suppress the acute lung injury or acute respiratory distress syndrome has been used, many specific therapies have not proved beneficial, such as mortality reduction ${ }^{4}$.

Statins are competitive inhibitors of 3-hydroxy-3methylglutaryl coenzyme A reductase resulting in a reduced cholesterol biosynthesis ${ }^{5}$. Besides, statins exert pleiotropic effects through reductions of pro-inflammatory cellular signaling ${ }^{5}$, oxidative stress ${ }^{6}$ and leukocyte interaction with endothelial cells ${ }^{7}$. These anti-inflammatory effects of statins have been reported in ischemia and reperfusion ${ }^{6}$ and extracorporeal membrane oxygenation in rat models ${ }^{8}$, as well as in lung transplanted patients ${ }^{9}$.

In the present study, we have investigated the beneficial effects of simvastatin in rats undergoing OLV followed by lung reexpansion ${ }^{10}$, evaluating the pulmonary and systemic inflammatory processes, as well as hemodynamic and gasometric parameters. The beneficial of simvastatin in OLV-induced lung injury after reoxygenation may place statins as a potential prophylactic therapy in this clinical condition.

\section{Methods}

The experiments were performed in accordance to the Guide for the Care and Use of Laboratory Animals with approval of the Ethics Committee on Experimental Animal Research CEEA-IB-UNICAMP (protocol number: 1610-1).

Male Wistar rats $(\mathrm{n}=30)$ weighing between 310 and 380 $\mathrm{g}$ were used. Animals were allocated randomly into 3 groups: (i) Control group with no manipulation (Control; $\mathrm{n}=12$ ), (ii) 1 - $\mathrm{h}$ OLV followed 1-h two-lung ventilation (OLV; $\mathrm{n}=9$ ) and (iii) 1 -h OLV followed 1-h two-lung ventilation in rats previously treated with $40 \mathrm{mg} / \mathrm{kg}$ of simvastatin (BioLab Farmacêutica Ltda, SP-Brazil) through oral gavage for 21 days before the surgical procedure
$(\mathrm{OLV}+$ Simvastatin; $\mathrm{n}=9)$

All animals were anesthetized with isoflurane 5\%, tracheostomized and ventilated with pressure-controlled ventilator

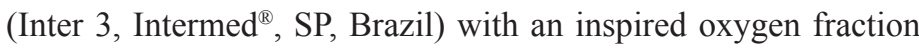
$\left(\mathrm{FiO}_{2}\right)$ of $60 \%$, respiratory rate $(\mathrm{RR})$ of 80 breaths per min, positive end expiratory pressure (PEEP) of $2 \mathrm{cmH}_{2} \mathrm{O}$ and inspiratory pressure (IP) of $20 \mathrm{cmH}_{2} \mathrm{O}$, as previous described ${ }^{11}$. The left femoral vein was dissected, and used for pancuronium bromide in bolus (1 mg/kg.h) administration. The same vein was used for administration of human albumin labeled with Iodine $\left({ }^{125} \mathrm{I}\right)$ and for fluid replacement, keeping a $0.9 \%$ saline solution infusion (1 $\mathrm{mL} / \mathrm{h})$. All animals were operated on a heated blanket with rectal temperature controlled between 36.0 to $37.5^{\circ} \mathrm{C}$.

The left carotid artery was cannulated and maintained with $0.9 \%$ heparinized saline $(5 \mathrm{UI} / \mathrm{mL})$ to obtain the blood arterial samples for gasometric analysis. The following gasometric parameters were evaluated: $\mathrm{pH}, \mathrm{PaO}_{2}, \mathrm{PaCO}_{2}, \mathrm{HCO}_{3}^{-}, \mathrm{BE}$ and $\mathrm{SO}_{2}$.

A right parasternal thoracotomy with ipsilateral anterolateral extension was performed. To establish the OLV protocol, all animals underwent atraumatic right lung mobilization, with clamping of the right main bronchus, keeping bilateral pulmonary perfusion and left lung ventilation. During OLV, no modification on the previously established ventilator parameters was performed, and animals remained under the same ventilatory condition. The clamp was maintained during $60 \mathrm{~min}$ and a new blood sample for gasometric analysis at the final minute of the OLV was collected.

In both groups, immediately after clamp removal, lung recruitment maneuver was performed through a sustained inflation at $30 \mathrm{cmH}_{2} \mathrm{O}$ during $30 \mathrm{sec}^{12}$, after which the ventilator parameters were adjusted to the previous values and remained unchanged during $60 \mathrm{~min}$. At the end of this period, a third arterial sample for gases analysis was performed and, immediately thereafter, rats received $5 \%$ isoflurane concentration and were sacrificed by exsanguination.

\section{Measurement of inflammatory markers in serum}

A midline laparotomy to collect blood from the abdominal aorta was performed. The blood collected was processed and the serum was stored for posterior measurements of levels of C-reactive protein CRP (CRP) (Chemicon ${ }^{\circledR}$ International, CA, USA), TNF- $\alpha$, IL-1 $\beta$, IL-6 and IL-10 (R\&D Systems ${ }^{\circledR}$, MN, USA) using commercial kits, according to the manufacturer's instructions.

Before removing the heart-lung block from the thoracic 
cavity, the lungs of all animals were perfused with $40 \mathrm{~mL}$ of $0.9 \%$ saline solution from a height of $20 \mathrm{~cm}$ through cannulation of the pulmonary artery, followed by opening the left atrium for effluent drainage. After perfusion, the lungs were removed and subjected to analyze of inflammatory markers through quantification of the myeloperoxidase (MPO) activity and pulmonary protein extravasation (as detailed below).

\section{Lung myeloperoxidase activity}

Therightand left lungs were removed and separately placed in a test tube in the presence of $0.5 \%$ hexadecyltrimethylammonium bromide in $50 \mathrm{mM}$ potassium phosphate buffer ( $\mathrm{pH}$ 6.0). The tissue samples were homogenized and centrifuged for $2 \mathrm{~min}$ at $12.000 \mathrm{~g}$, and the supernatant collected. MPO activity assay was performed using a microplate spectrophotometer (Spectra Max 34; Molecular Devices, Sunnyvale, CA, USA). Briefly, the assay consisted of mixing a $5 \mu \mathrm{L}$ sample with $200 \mu \mathrm{L}$ of o-dianisidine solution $(0.167 \mathrm{mg} / \mathrm{mL}$ o-dianisidine dihydrochoride; $0.0005 \%$ hydrogen peroxide) prior to reading the plate. The changes in absorbance were measured at $460 \mathrm{~nm}$ recorded at intervals of 15 sec during $10 \mathrm{~min}$. MPO activity was expressed as units of enzyme activity per milligram of tissue (UMPO)/mg. One unit of MPO was defined the enzyme amount that degrades one micromole of peroxide/min at $25^{\circ} \mathrm{C}^{13}$.

\section{Pulmonary protein extravasation}

Pulmonary protein extravasation was measured by means of intravenously injected ${ }^{125} \mathrm{I}$ serum albumin $(2.5 \mu \mathrm{Ci} /$ $\mathrm{kg}$ ) accumulation. The radioactivity present in blood samples and lung tissue was quantified separately for one minute each in a $\gamma$-counter ${ }^{14}$. The protein extravasation was expressed as the volume (in microlitres) of serum accumulated in the lung derived from the total count in $1 \mathrm{~mL}$ serum.

\section{Statistical analysis}

Statistical analysis was performed using Software GraphPad Prism 5.00. All variables were tested for normal distribution. Parametric data were presented as means \pm standard error of the mean (SEM), with p-values resulted of the comparison between groups by applying $t$-test or ANOVA followed by Bonferroni post-test. Differences were considered to be statistically significant for all procedures if $\mathrm{p}<0.05$.

\section{Results}

Activity of myeloperoxidase (MPO) in lung tissues

The MPO activity in the right and left lungs after OLV was significantly greater $(24.5 \pm 0.9$ and $25.9 \pm 1.0 \mu \mathrm{L} / \mathrm{g}$, respectively) compared with animals under no manipulation (18.7 \pm 6.0 and $17.2 \pm 4.4 \mu \mathrm{L} / \mathrm{g}$, respectively). Simvastatin treatment significantly reduced the MPO activity in the left lung after OLV $(\mathrm{p}=0.044)$, without affecting the increased MPO in the right lungs $(\mathrm{p}=0.3530$; Figure 1).

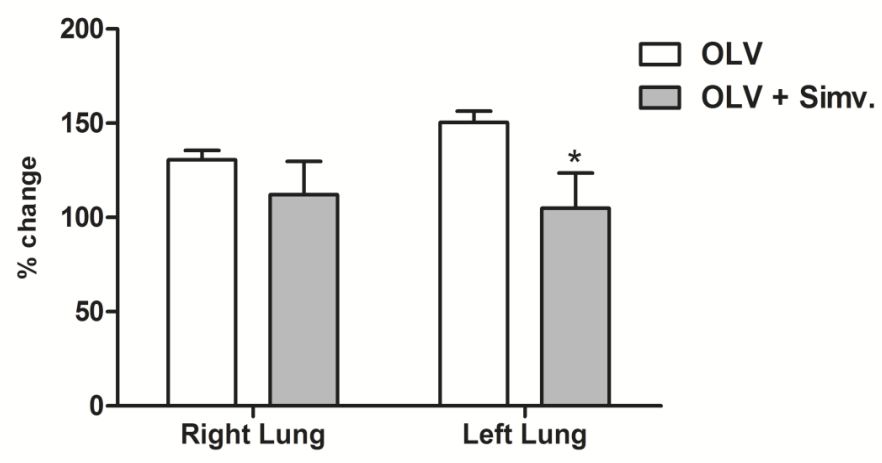

FIGURE 1 - Simvastatin (Simv) treatment prior to OLV reduced the myeloperoxidase (MPO) activity in the left lung tissue after one-lung ventilation $(\mathrm{OLV})$ in rats.

Measurement of MPO activity was performed in the right and left lungs of rats from the control, OLV and OLV+Simv groups. Results are shown as mean of \% change \pm SEM $(n=9)$, calculated based on the control group mean value. ${ }^{*} \mathrm{p}=0.044$ (Student's $t$ test) compared to the left lung of OLV group.

\section{Pulmonary microvascular permeability}

The pulmonary protein extravasation in the right and left lungs after OLV $(173.8 \pm 27.7$ and $121.5 \pm 20.4 \mu \mathrm{L} / \mathrm{g}$, respectively $)$ differ significantly from animals under no manipulation (50.1 \pm 3.6 and $47.6 \pm 3.8 \mu \mathrm{L} / \mathrm{g}$, respectively). Prolonged treatment with simvastatin did not change significantly the pulmonary protein extravasation seen in the right and left lungs after OLV (222.9 \pm 19.3 and $176.9 \pm 24.4 \mu \mathrm{L} / \mathrm{g}$, respectively) (Figure 2). 


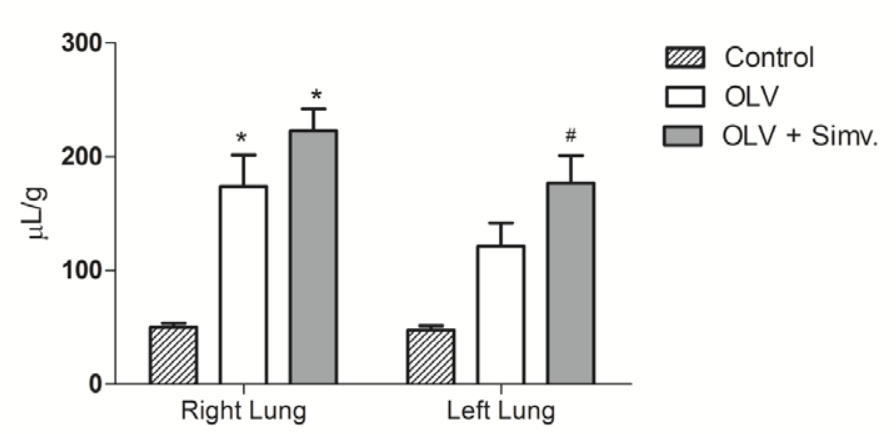

FIGURE 2 - Pulmonary protein extravasation. Data are represented as mean and SEM, with analysis performed by ANOVA followed by Bonferroni post-test $\left({ }^{*} \mathrm{p}<0.05\right.$ versus right lung of Control group; \# $\mathrm{p}<0.05$ versus left lung of Control group).

\section{Serum levels of $I L-1 \beta, T N F-\alpha, I L-10, I L-6$ and $C R P$}

The IL-1 $\beta$, TNF- $\alpha$ and IL-10 levels remained below the detection limit in all groups $(62.5 \mathrm{pg} / \mathrm{mL})$. The IL-6 and CRP levels were markedly higher in OLV group $(\mathrm{p}<0.05)$, but simvastatin treatment failed to affect the production of both mediators (Table $1)$.

TABLE 1 - Serum levels of IL-6 and CRP.

\begin{tabular}{cccc} 
& Control & OLV & $\begin{array}{c}\text { OLV + } \\
\text { Simvastatin }\end{array}$ \\
\hline IL-6, $\mathbf{n g} / \mathbf{m L}$ & ND & $4.0 \pm 1.4^{*}$ & $5.1 \pm 1.5^{*}$ \\
CRP, $\mathbf{~ m g / m L ~}$ & $0.68 \pm 0.07$ & $5.1 \pm 0.3^{*}$ & $4.8 \pm 0.4^{*}$ \\
\hline
\end{tabular}

Data are presented as mean \pm SEM from nine rats. * $\mathrm{p}<0.05$ compared with Control Group (ANOVA followed by Bonferroni post test). OLV, one-lung ventilation. ND, not detected.

\section{Gasometric parameters}

Figure 2 shows the $\mathrm{PaO}_{2}$ in the experimental groups. During OLV, a significant decrease in $\mathrm{PaO}_{2}$, as observed in both non-treated and simvastatin-treated rats, was observed. $\mathrm{PaO}_{2}$ values returned to baseline after lung re-expansion period and reestablishment of bilateral ventilation. No differences were observed between non-treated and simvastatin-treated rats (Figure 3).

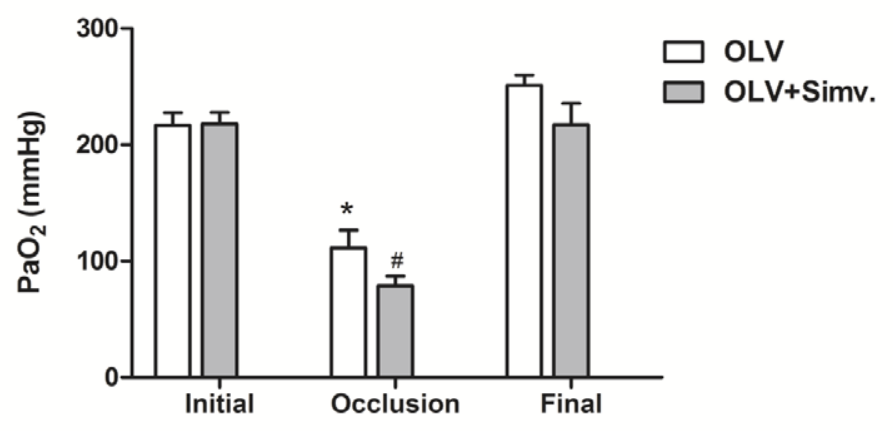

FIGURE 3 - $\mathrm{PaO}_{2}$ values at the beginning of the protocol (initial), and after 1-h of OLV (bronchial occlusion) and subsequently to 1-h of twolung ventilation (final). Data represent mean \pm SEM for nine rats. ${ }^{*} \mathrm{p}<0.05$ versus initial and final measurements in OLV group; $\# \mathrm{p}<0.05$ versus initial and final measurements in OLV+Simv group (ANOVA followed by Bonferroni post hoc test). OLV, one-lung ventilation.

As shown in Table 2, no gasometric parameter $(\mathrm{pH}$, $\mathrm{PaCO}_{2}, \mathrm{HCO}_{3}^{-}, \mathrm{BE}$ and $\mathrm{SO}_{2}$ ) showed significantly changes in an equal period when compared the groups that received or not simvastatin previous treatment.

TABLE 2 - Blood gas analysis obtained in different stages of OLV followed by TLV.

\begin{tabular}{|c|c|c|c|}
\hline & & OLV & OLV + Simvastatin \\
\hline \multirow[t]{3}{*}{$\mathbf{p H}$} & Initial & $7.41 \pm 0.01$ & $7.44 \pm 0.01$ \\
\hline & Occlusion & $7.27 \pm 0.04$ & $7.29 \pm 0.04 *$ \\
\hline & Final & $7.40 \pm 0.01$ & $7.35 \pm 0.05$ \\
\hline \multirow[t]{3}{*}{$\mathrm{PaCO}_{2}(\mathrm{mmHg})$} & Initial & $38.98 \pm 0.96$ & $35.33 \pm 1.81$ \\
\hline & Occlusion & $59.31 \pm 8.79$ & $56.63 \pm 7.47$ \\
\hline & Final & $37.65 \pm 1.17$ & $48.93 \pm 10.53$ \\
\hline \multirow[t]{3}{*}{$\mathrm{HCO}_{3}^{-}(\mathrm{mmol} / \mathrm{L})$} & Initial & $24.76 \pm 0.52$ & $23.55 \pm 0.79$ \\
\hline & Occlusion & $24.99 \pm 0.91$ & $24.96 \pm 0.47$ \\
\hline & Final & $22.50 \pm 0.50$ & $23.71 \pm 1.08$ \\
\hline \multirow[t]{3}{*}{ BE (mmol/L) } & Initial & $0.62 \pm 0.64$ & $0.39 \pm 0.59$ \\
\hline & Occlusion & $\begin{array}{c}-2.67 \pm \\
0.69 *\end{array}$ & $-2.23 \pm 0.92$ \\
\hline & Final & $-1.46 \pm 0.49$ & $-1.844 \pm 0.84$ \\
\hline \multirow[t]{3}{*}{$\mathrm{SO}_{2}(\%)$} & Initial & $99.56 \pm 0.06$ & $99.59 \pm 0.05$ \\
\hline & Occlusion & $95.41 \pm 1.21$ & $91.23 \pm 2.28 *$ \\
\hline & Final & $99.73 \pm 0.02$ & $99.39 \pm 0.21$ \\
\hline
\end{tabular}

Data are presented as mean \pm SEM for nine rats. ${ }^{*} \mathrm{p}<$ 0.05 versus initial (ANOVA followed by Bonferroni post hoc test). OLV, one-lung ventilation. 


\section{Discussion}

In the present study, we show that pre-operative treatment with simvastatin reduces remote pulmonary neutrophil infiltration, without affecting any other local or systemic parameter.

Neutrophils are the earliest cells recruited to tissues followed an inflammatory insult. These cells are an important source of reactive oxygen species, with clinical and experimental evidences indicating that these cells greatly contribute to the acute lung injury and acute respiratory distress syndrome ${ }^{4}$. Statins have been shown to have a potent anti-inflammatory effect in acute local inflammation, as a consequence of its inhibitory effect on polymorphonuclear leucocyte infiltration ${ }^{15}$. Accordingly, in our study, treatment with simvastatin significantly reduced the MPO activity in the left lung, which remained continuously ventilated throughout the experiment. Previous studies have shown that OLV can be consider a mechanism that increases lung microvascular permeability leading to edema formation in humans after lung resections ${ }^{16}$, with the same behavior reproduced in experimental studies using different animal species as well as diverse in the bronchial occlusion time ${ }^{10,17}$. However, no studies exist evaluating the effects of statins on the increased microvascular permeability in models lung re-expansion after bronchial occlusion. In the present study, OLV significantly increased the pulmonary vascular permeability after lung re-expansion; however, simvastatin pretreatment failed to affect the increased permeability. Simvastatin promotes a significant reduction in pulmonary vascular permeability in other models of pulmonary inflammation such as lung ischemia and reperfusion ${ }^{6}$. Whether these conflicting findings reflect differences in the dose of simvastatin used and experimental paradigm requires additional studies.

Besides the local inflammatory response, its systemic repercussion was also investigated through analysis of serum inflammatory mediators. TNF- $\alpha$, an early proinflammatory cytokine, activates other pro-inflammatory cytokines such as IL$1 \beta$ and IL-6. TNF- $\alpha$ and IL- $1 \beta$ activates the inflammatory cascade by producing many cytokines and chemokines and release of endothelial adhesion molecules ${ }^{18}$. In our study, the levels of the pro-inflammatory cytokines TNF- $\alpha$, IL- $1 \beta$ and IL-10 remained below the limit of detection, which is suggestive that the $1-\mathrm{h}$ period of OLV followed by an equal period of bilateral ventilation caused only a local pulmonary inflammatory response without systemic repercussion. This is consistent with a previous study in pigs undergoing a similar protocol ${ }^{17}$. We may also consider that volatile anesthetics reduces inflammatory responses in ischemia and reperfusion models due to inhibition of the TNF- $\alpha$ release ${ }^{19}$.
The cytokine IL-6 was systemically detected, but no differences between untreated and simvastatin-treated groups were observed. Therefore, it is unlikely that reduction of pulmonary neutrophil infiltration by simvastatin reflects enhanced levels of IL-6. The IL-6 response to statin therapy has been controversial in the literature $^{20}$. The CRP levels were largely greater in OLV group compared with control animals, but simvastatin failed to affect this increased production.

Finally, regarding the gasometric analysis, $\mathrm{pH}$ and $\mathrm{SO}_{2}$ values decreased significantly at the end of the OLV period compared with baseline measurements in the simvastatin group. A similar response was verified in $\mathrm{BE}$ data measured in the untreated group. Considering that no intervention was performed to control the respiratory acidosis that followed the occlusion period, the kidney function was not sufficient to compensate for the homeostase during the OLV. However, in all cases a regression to the baseline parameters was observed after a relatively short period of bilateral ventilation (1 hour). As seen for the first time, simvastatin treatment caused no protective effect in any evaluated gasometric parameter during OLV. A previous study in rats undergoing cardiopulmonary bypass showed a large protective effect by simvastatin on the decreased $\mathrm{PaO}_{2}$ values which was attributed to increased nitric oxide production after hypoxia ${ }^{8}$. However, it is worth mentioning that in the present model no severe hypoxic condition occurred, even during OLV.

\section{Conclusions}

In an experimental one-lung ventilation model preoperative treatment with simvastatin reduces remote neutrophil infiltration in the ventilated lung. Our findings suggest that simvastatin may be of therapeutic value in OLV-induced pulmonary inflammation deserving clinical investigations.

\section{References}

1. Olivant Fisher A, Husain K, Wolfson MR, Hubert TL, Rodriguez E, Shaffer TH, Shaffer TH, Theroux MC. Hyperoxia during one lung ventilation: inflammatory and oxidative responses. Pediatr Pulmonol. 2012;47(10):979-86.

2. Tsai JA, Lund M, Lundell L, Nilsson-Ekdahl K. One-lung ventilation during thoracoabdominal esophagectomy elicits complement activation. J Surg Res. 2009;152(2):331-7.

3. Gothard J. Lung injury after thoracic surgery and one-lung ventilation. Curr Opin Anaesthesiol. 2006;19(1):5-10.

4. Ware LB, Matthay MA. The acute respiratory distress syndrome. N Engl J Med. 2000;342(18):1334-49.

5. Hothersall E, McSharry C, Thomson NC. Potential therapeutic role for statins in respiratory disease. Thorax. 2006;61(8):729-34.

6. Naidu BV, Woolley SM, Farivar AS, Thomas R, Fraga C, Mulligan 
MS. Simvastatin ameliorates injury in an experimental model of lung ischemia-reperfusion. J Thorac Cardiovasc Surg. 2003;126(2):4829.

7. Pruefer D, Scalia R, Lefer AM. Simvastatin inhibits leukocyteendothelial cell interactions and protects against inflammatory processes in normocholesterolemic rats. Arterioscler Thromb Vasc Biol. 1999;19(12):2894-900.

8. Shao H, Shen Y, Liu H, Dong G, Qiang J, Jing H. Simvastatin suppresses lung inflammatory response in a rat cardiopulmonary bypass model. Ann Thorac Surg. 2007;84(6):2011-8.

9. Johnson BA, Iacono AT, Zeevi A, McCurry KR, Duncan SR. Statin use is associated with improved function and survival of lung allografts. Am J Respir Crit Care Med. 2003;167(9):1271-8.

10. Leite CF, Calixto MC, Toro IFC, Antunes E, Mussi RK. Characterization of Pulmonary and systemic inflammatory responses produced by lung re-expansion after one-lung ventilation. J Cardiothorac Vasc Anesth. 2012;26(3):427-32.

11. Leite CF, Toro IFC, Antunes E, Mussi RK. Standardization of a method of prolonged thoracic surgery and mechanical ventilation in rats to evaluate local and systemic inflammation. Acta Cir Bras. 2011;26(1):38-43.

12. de Carvalho MEP, Dolhnikoff M, Meireles SI, Reis LFL, Martins MA, Deheinzelin D. Effects of overinflation on procollagen type III expression in experimental acute lung injury. Crit Care. 2007;11(1):R23.

13. Bradley PP, Priebat DA, Christensen RD, Rothstein G. Measurement of cutaneous inflammation: estimation of neutrophil content with an enzyme marker. J Invest Dermatol. 1982;78(3):206-9.

14. Bhabra MS, Hopkinson DN, Shaw TE, Onwu N, Hooper TL. Controlled reperfusion protects lung grafts during a transient early increase in permeability. Ann Thorac Surg. 1998;65(1):187-92.

15. Nezić L, Skrbić R, Dobrić S, Stojiljković MP, Jaćević V, Satara SS, Milovanović ZA, Stojaković N. Simvastatin and indomethacin have similar anti-inflammatory activity in a rat model of acute local inflammation. Basic Clin Pharmacol Toxicol. 2009;104(3):185-91.

16. Padley SPG, Jordan SJ, Goldstraw P, Wells AU, Hansell DM. Asymmetric ARDS following pulmonary resection: CT findings initial observations. Radiology. 2002;223(2):468-73.

17. Yin K, Gribbin E, Emanuel S, Orndorff R, Walker J, Weese J, Fallahnejad M. Histochemical alterations in one lung ventilation. J Surg Res. 2007;137(1):16-20.

18. Liu D, Zeng BX, Zhang SH, Yao SL. Rosiglitazone, an agonist of peroxisome proliferator-activated receptor gamma, reduces pulmonary inflammatory response in a rat model of endotoxemia. Inflamm Res. 2005;54(11):464-70.

19. Liu $\mathrm{R}$, Ishibe $\mathrm{Y}, \mathrm{Ph} \mathrm{D}$, Ueda $\mathrm{M}$. Isoflurane-sevoflurane adminstration before ischemia attenuates ischemia-reperfusion-induced injury in isolated rat lungs. Anesthesiology. 2000;92(3):833-40.

20. Pleiner J, Schaller G, Mittermayer F, Zorn S, Marsik C, Polterauer S, Kapiotis S, Wolzt M. Simvastatin prevents vascular hyporeactivity during inflammation. Circulation. 2004;110(21):3349-54.

\section{Correspondence:}

Ricardo Kalaf Mussi

Departamento de Cirurgia - Faculdade de Ciências Médicas

UNICAMP

Rua Tessália Vieira de Camargo, 126

13083-887 Campinas - SP Brasil

Tel.: (55 19)3521-9441

Fax: (55 19)3521-8043

rkm@unicamp.br

Received: December 18, 2012

Review: February 20, 2013

Accepted: March 19, 2013

Conflic of interest: none

Financial sources: Coordination of Improvement for Higher Academic Staff (CAPES) and Sao Paulo Research Foundation (FAPESP)

${ }^{1}$ Research performed at Laboratory of Inflammation, Department of Pharmacology, Faculty of Medical Sciences, State University of Campinas (UNICAMP), Brazil. Part of Master degree thesis, Postgraduate Program in Surgical Sciences, UNICAMP. Tutor: Prof. Ricardo Kalaf Mussi. 